\begin{tabular}{|c|l|}
\hline Title & Spin density wave in the strongly dimerized quasi-one dimensional organic conductor (DMET-TTF)(2)A uBr2 \\
\hline Author(s) & $\begin{array}{l}\text { Iida, Youhei; Sawada, Masashi; Sasaki, Y oshiaki; Tsuchiya, Tomoaki; Minamidate, Takaaki; Matsunaga, Noriaki; } \\
\text { Kawamoto, A tsushi; Nomura, Kazushige }\end{array}$ \\
\hline Citation & $\begin{array}{l}\text { Physical Review B, 104(18), 184409 } \\
\text { https://doi.org/10.1103/PhysRevB.104.184409 }\end{array}$ \\
\hline Issue Date & 2021-11-04 \\
\hline Doc URL & http://hdl.handle.net/2115/83511 \\
\hline Rights & @2021 A merican Physical Society \\
\hline Type & article \\
\hline File Information & PhysRevB.10418_184409.pdf \\
\hline
\end{tabular}

Instructions for use 


\title{
Spin density wave in the strongly dimerized quasi-one-dimensional organic conductor (DMET-TTF $)_{2} \mathrm{AuBr}_{2}$
}

\author{
Youhei Iida $\odot,{ }^{1}$ Masashi Sawada, ${ }^{1}$ Yoshiaki Sasaki, ${ }^{1}$ Tomoaki Tsuchiya, ${ }^{1}$ Takaaki Minamidate, ${ }^{1,2}$ \\ Noriaki Matsunaga $\odot,{ }^{1, *}$ Atsushi Kawamoto $\odot,{ }^{1}$ and Kazushige Nomura ${ }^{1}$ \\ ${ }^{1}$ Department of Physics, Hokkaido University, Sapporo 060-0810, Japan \\ ${ }^{2}$ Department of Applied Physics, Tokyo University of Science, Tokyo 125-8585, Japan
}

(Received 7 June 2021; revised 17 September 2021; accepted 25 October 2021; published 4 November 2021)

\begin{abstract}
At ambient pressure, studies of resistivity, magnetic susceptibility, and carbon-13 nuclear magnetic resonance (NMR) were conducted on the quasi-one-dimensional organic conductor (DMET-TTF) ${ }_{2} \mathrm{AuBr}_{2}$. Resistivity measurements showed a broad minimum at approximately $160 \mathrm{~K}$, and the insulator behavior below this temperature and magnetic susceptibility results revealed a dip structure at $22 \mathrm{~K}$. At the same temperature, a sharp peak in the temperature dependence of $T_{1}^{-1}$ associated with the antiferromagnetic (AFM). transition was found, along with drastic splitting of the NMR spectra, indicating a commensurate AFM structure. The amplitude of the magnetic moments was determined to be $0.06 \mu_{B} /$ molecule from the hyperfine coupling constant tensor and the angular dependence of the internal field in the AFM phase. The small magnetic moment signifies the AFM nesting type, i.e., commensurate spin density wave. An antiferromagnetic ordering of ( $\uparrow \downarrow \downarrow)$ along the one-dimensional chain is expected from the $2 k_{\mathrm{F}}$ instability. This behavior can be explained by the strong dimerization of the one-dimensional DMET-TTF chain.
\end{abstract}

DOI: 10.1103/PhysRevB.104.184409

\section{INTRODUCTION}

The study of low-dimensional electronic systems with a strong electronic correlation has been an attractive topic in the field of condensed matter physics. (TMTCF $)_{2} X(C=$ S; TMTTF: tetramethyltetrathiafulvalene, $C=\mathrm{Se}$; TMTSF: tetramethyltetraselenafulvalene) salts are well known as the most investigated quasi-one-dimensional (Q1D) organic systems, with a complex phase diagram exhibiting charge ordering (CO), charge localization (CL), spin Peierls (SP), antiferromagnetism (AFM), spin density wave (SDW), as well as metallic and superconducting phases depending on pressure or anions. (TMTSF) ${ }_{2} \mathrm{PF}_{6}$ at ambient pressure undergoes an incommensurate SDW (IC-SDW) transition at $12 \mathrm{~K}$ [1] and the superconducting phase appears at $0.6 \mathrm{GPa}$. (TMTTF $)_{2} \mathrm{Br}$ is located at a lower-pressure region than (TMTSF) ${ }_{2} \mathrm{PF}_{6}$ in the generalized phase diagram of (TMTCF $)_{2} X$ [2]. The ground state in (TMTTF $)_{2} \mathrm{Br}$ has been reported as commensurate SDW (C-SDW) [3] with a $(\uparrow \circ \downarrow \circ)$ type magnetic structure $[4,5]$. To investigate the role of the dimensionality, electronic correlation, and dimerization of the donor in the Q1D system, we focus on the us-DMETSTF [dimethyl(ethylenedithio)diselenadithiafulvalene, abbreviated as DMET-STF hereafter] where "us" denotes the molecule's unsymmetrical shape due to the positioning of $\mathrm{Se}$ atoms as shown in Fig. 1(a) and DMET-TTF [dimethyl(ethylenedithio)tetrathiafulvalene] salts with linear anions such as $\mathrm{Au}(\mathrm{CN})_{2}, \mathrm{AuI}_{2}, \mathrm{AuBr}_{2}$, and $\mathrm{I}_{3}$; both have a Q1D Fermi surface in the case of the 2:1 stoichiometry of the donor and anion.

*mat@phys.sci.hokudai.ac.jp
The DMET-STF donor is the first unsymmetrical donor that gives an organic superconductor in (DMET-STF $)_{2}$ $\mathrm{Au}(\mathrm{CN})_{2}$ [6]. An isostructural salt (DMET-STF) ${ }_{2} \mathrm{AuI}_{2}$ becomes SDW at ambient pressure and a superconductor at $0.5 \mathrm{GPa}$ [7], (DMET-STF $)_{2} \mathrm{I}_{3}$ shows a superconducting transition at ambient pressure [8], and (DMET-STF) ${ }_{2} \mathrm{AuBr}_{2}$ exhibits metallic behavior in the entire temperature region [9]. Additionally, (DMET-TTF $)_{2} \mathrm{I}_{3}$ presents a metal-insulator (SDW) transition at $40 \mathrm{~K}[10]$. These results suggest that DMET-TTF salts are located at a lower-pressure region than DMET-STF salts. Moreover, the DMET families with linear anions can be described by the universal phase diagram depending on the pressure or anions such as TMTCF families. However, the DMET donors are alternately stacked unsymmetrical donors, and the normal to the molecular plane is largely tilted from the stacking axis. Therefore, DMET families are expected to have a stronger dimerization effect than the TMTCF families.

${ }^{13} \mathrm{C}$ NMR is a robust method to examine the electronic state of organic conductors. In TMTCF families, one of us synthesized the single-site enriched donor in the central carbon, free from the influence of the Pake doublet. The carbon site in the central double bond is crystallographically independent in the DMET families with linear anions, whereas nonequivalent carbon sites exist in the central double bond in TMTCF families, which are called inner and outer sites. Therefore, ${ }^{13} \mathrm{C}$-NMR measurements in the DMET families are powerful tools for examining magnetic and electronic structures. To elucidate the role of dimensionality, electronic correlation, and donor dimerization in the Q1D system, we synthesized the single-site enriched (DMET-TTF $)_{2} \mathrm{AuBr}_{2}$ [expected to be located at a lowerpressure region than (DMET-STF $\left.)_{2} \mathrm{AuBr}_{2}\right]$, and measured 


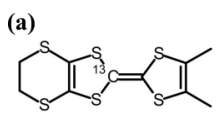

DMET-TTF

(b)
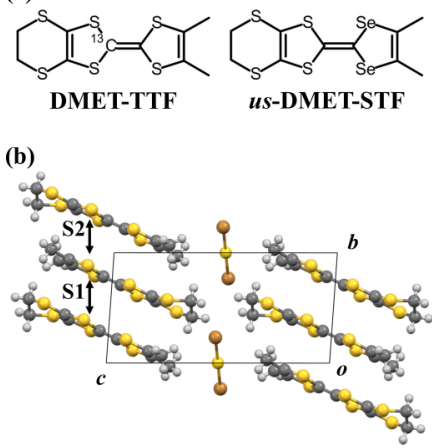

(c)

(d)

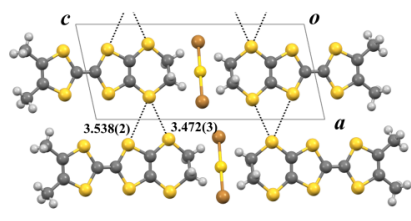

FIG. 1. (a) Structure of ${ }^{13} \mathrm{C}$-enriched DMET-TTF and us-DMETSTF molecules. (b) Crystal structure of (DMET-TTF) $)_{2} \mathrm{AuBr}_{2}$, viewed along the $a$ axis. (c) DMET-TTF overlaps in the molecular plane along the normal axis. (d) Crystal structure of (DMET-TTF $)_{2} \mathrm{AuBr}_{2}$, viewed along the $b$ axis.

resistivity, magnetic susceptibility, and ${ }^{13} \mathrm{C}$ NMR at ambient pressure.

\section{EXPERIMENTS}

As shown in Fig. 1(a), we prepared single-site ${ }^{13} \mathrm{C}$-substituted DMET-TTF molecules using the crosscoupling method in the synthetic route of BEDT-TTF [bis(ethylenedithio)tetrathiafulvalene] [11,12]. The donor molecule was synthesized by cross-coupling between 4,5-ethylenedithio-1,3-dithiole-2-one and 4,5-dimethyl-1,3dithiol-2-selone in triethyl phosphite and purified by column chromatography. Furthermore, the standard electrochemical oxidation process was used to prepare single crystals of (DMET-TTF $)_{2} \mathrm{AuBr}_{2}$.

The crystal structure of (DMET-TTF $)_{2} \mathrm{AuBr}_{2}$ was determined by $\mathrm{x}$-ray diffraction measurement using a Rigaku R-AXIS RAPID diffractometer with Mo $K \alpha$ radiation. Moreover, the crystal structure was solved with SIR2002 [13] and refined with SHELX97 [14]. Mercury [15] was used to calculate the intermolecular and interplanar distance and visualize the crystal structure. Transfer integrals, band dispersion, and the Fermi surface are calculated using the tight-binding approximation [16]. The molecular orbitals were calculated by the extended Hückel method with semiempirical parameters [17]. In this calculation, the $d$ orbitals in the $\mathrm{S}$ atoms were not taken into account.

Additionally, the $b$-axis resistivity was measured using a single crystal attached by gold wires with carbon paste and the sample dimensions were $0.17 \times 0.50 \times 0.12 \mathrm{~mm}^{3}$. The $b$-axis conductivity is approximately 50 times larger than the $a$-axis conductivity. At the low resistance region, we used the fourprobe method. A Keithley 220 current source supplied the current and the voltage was measured by an Agilent 34420A nano volt/micro-ohm meter. At the high resistance region, we measured two-terminal resistance using a Keithley 6517A meter.

Furthermore, the susceptibility of a powdered sample of (DMET-TTF $)_{2} \mathrm{AuBr}_{2}$ was measured under a magnetic field of $2 \mathrm{~T}$ using a magnetic property measurement system superconducting quantum interference device (MPMS3 SQUID) magnetometer (Quantum Design). The sample's mass was $4.9 \mathrm{mg}$ and the samples were wrapped in aluminum foil and affixed to a straw. The susceptibility of powdered DMET-TTF, $\mathrm{TBA} \cdot \mathrm{AuBr}_{2}$, and TBA $\cdot \mathrm{Br}$ was similarly measured at $300 \mathrm{~K}$.

${ }^{13} \mathrm{C}$-NMR experiments were performed for a single crystal of single-site ${ }^{13} \mathrm{C}$-substituted (DMET-TTF) $)_{2} \mathrm{AuBr}_{2}$ in a magnetic field of $6 \mathrm{~T}$. Moreover, the crystal orientation was determined by x-ray diffraction, and spectra were obtained by the fast Fourier transformation of the echo signal with $\pi / 2-\pi$ where the $\pi / 2$ pulse width was 3 or $5 \mu \mathrm{s}$. The spin-lattice relaxation rate $T_{1}$ was determined by the saturation recovery method.

\section{RESULTS AND DISCUSSION}

\section{A. Crystal structure}

For (DMET-TTF) ${ }_{2} \mathrm{AuBr}_{2}$, the space group is $P \overline{1}$ and the lattice parameters are $a=6.6765(6) \AA, b=7.6247(5)$ $\AA, c=15.4366(11) \AA, \alpha=91.192(2)^{\circ}, \beta=101.783(4)^{\circ}$, $\gamma=103.236(4)^{\circ}, V=746.94(10) \AA^{3}$, and $Z=2$ at ambient pressure. The crystals are platelike, and the shortest axis corresponds to the $c$ axis. As a result of selenium substitution to sulfur, the unit cell volume of (DMET-TTF) ${ }_{2} \mathrm{AuBr}_{2}$ is smaller than that of (DMET-STF) ${ }_{2} \mathrm{AuBr}_{2}$. Angles $\alpha, \beta$, and $\gamma$ are nearly equivalent and $a, b$, and $c$ are smaller than those of (DMET-STF) ${ }_{2} \mathrm{AuBr}_{2}$, as listed in Table I, suggesting that (DMET-TTF) $)_{2} \mathrm{AuBr}_{2}$ and (DMET-STF) ${ }_{2} \mathrm{AuBr}_{2}$ [18] are isostructures.

Figure 1(b) shows the crystal structure of (DMET-TTF) $)_{2} \mathrm{AuBr}_{2}$. Along the $b$ axis, DMET-TTF molecules are stacked. The molecular plane's normal axis is inclined $23.2^{\circ}$ away from the stacking axis. As illustrated in Fig. 1(c), S1 and S2 indicate two forms of molecular overlap. The interplanar distances are $3.46 \AA$ for $\mathrm{S} 1$ and $3.56 \AA$ for $\mathrm{S} 2$, where the molecular plane is determined by the central carbon and the sulfur on the fulvalene structure. Two shorter contacts exist between the adjacent columns, as shown by the dotted lines in Fig. 1(d). The overlap integrals in the $c$ direction are insignificant because the anions and DMET-TTF molecule layers stack alternately.

\section{B. Band structure}

To discuss the electronic structure, we calculated the band structure of the Q1D salts. The overlap integrals in (DMET-TTF) $)_{2} \mathrm{AuBr}_{2}$ at ambient pressure are

TABLE I. The lattice parameters of (DMET-STF) $)_{2} \mathrm{AuBr}_{2}$ and (DMET-TTF) $)_{2} \mathrm{AuBr}_{2}$.

\begin{tabular}{|c|c|c|c|c|c|c|c|}
\hline & $a(\AA)$ & $b(\AA)$ & $c(\AA)$ & $\alpha(\mathrm{deg})$ & $\beta(\mathrm{deg})$ & $\gamma(\operatorname{deg})$ & $V\left(\AA^{3}\right)$ \\
\hline$(\mathrm{DMET}-\mathrm{STF})_{2} \mathrm{AuBr}_{2}$ [18] & $6.732(4)$ & $7.682(6)$ & $15.682(7)$ & $91.44(4)$ & $101.58(4)$ & $104.01(3)$ & $768.5(8)$ \\
\hline$(\mathrm{DMET}-\mathrm{TTF})_{2} \mathrm{AuBr}_{2}$ & $6.6765(6)$ & $7.6247(5)$ & $15.4366(11)$ & $91.192(2)$ & $101.783(4)$ & $103.236(4)$ & $746.94(10)$ \\
\hline
\end{tabular}




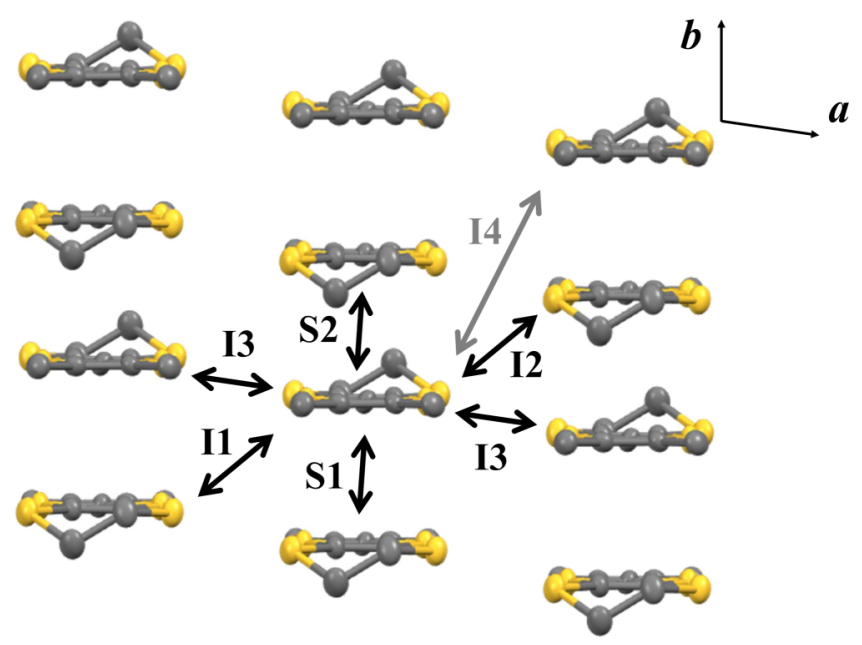

FIG. 2. Definition of the symbols for the overlap integrals of (DMET-TTF $)_{2} \mathrm{AuBr}_{2}$.

$S_{\mathrm{S} 1}=-0.01427, S_{\mathrm{S} 2}=-0.01112, S_{\mathrm{I} 1}=-0.00115, S_{\mathrm{I} 2}=$ -0.00187 , and $S_{\mathrm{I} 3}=0.00134$, where the symbols are defined in Fig. 2. Using the multiple-transverse-transfer model $[19,20]$, the overlap integrals along to stacking direction $S_{\mathrm{S}}$ and in the interstack direction $S_{\mathrm{I}}$ are calculated as $S_{\mathrm{S}}=$ 0.01269 and $S_{\mathrm{I}}=0.00110$, respectively. The anisotropy of overlap integrals is $S_{\mathrm{S}} / S_{\mathrm{I}}=11.5$, suggesting a Q1D electronic structure. The overlap integrals in (DMET-STF $)_{2} \mathrm{AuBr}_{2}$ and $(\mathrm{TMTTF})_{2} \mathrm{Br}$ were calculated using the same method and the results are also listed in Table II. The atomic coordinates were taken from the literature [18,21], and those of hydrogen in (DMET-STF $)_{2} \mathrm{AuBr}_{2}$ were determined geometrically using OLEX2 [22]. The calculation in (TMTTF) ${ }_{2} \mathrm{Br}$ reproduced previous results $[19,23]$. In the case of (DMET-STF) ${ }_{2} \mathrm{AuBr}_{2}$ [18], the overlap integrals are almost twice as large as those of (DMET-TTF $)_{2} \mathrm{AuBr}_{2}$ due to selenium substitution. As the value of $S_{\mathrm{S}} / S_{\mathrm{I}}$ of (DMET-TTF) ${ }_{2} \mathrm{AuBr}_{2}$ is slightly smaller than that of (TMTTF) $)_{2} \mathrm{Br}$, (DMET-TTF $)_{2} \mathrm{AuBr}_{2}$ is more two dimensional than (TMTTF) ${ }_{2} \mathrm{Br}$. As $S_{\mathrm{S}}$ is proportional to the bandwidth, the bandwidth of (DMET-TTF) ${ }_{2} \mathrm{AuBr}_{2}$ is narrower than that of (DMET-STF) ${ }_{2} \mathrm{AuBr}_{2}$ and almost the same in (TMTTF) $)_{2} \mathrm{Br}$. According to the two types of molecular overlap $\mathrm{S} 1$ and $\mathrm{S} 2$, the effective dimerization of the overlap integrals $S_{\mathrm{S} 2} / S_{\mathrm{S} 1}$ in (DMET-TTF) ${ }_{2} \mathrm{AuBr}_{2}$ is 0.78 , which is smaller than 0.9 of (TMTTF $)_{2} \mathrm{Br}$, suggesting that (DMET-TTF $)_{2} \mathrm{AuBr}_{2}$ is a strongly dimerized Q1D system, although this dimerization is not as large as that of a typical dimer Mott insulator.

Figure 3 shows the band dispersion and the Fermi surface in (DMET-TTF $)_{2} \mathrm{AuBr}_{2}$ using an extended Hückel tight-binding method. The upper antibonding band is effec-

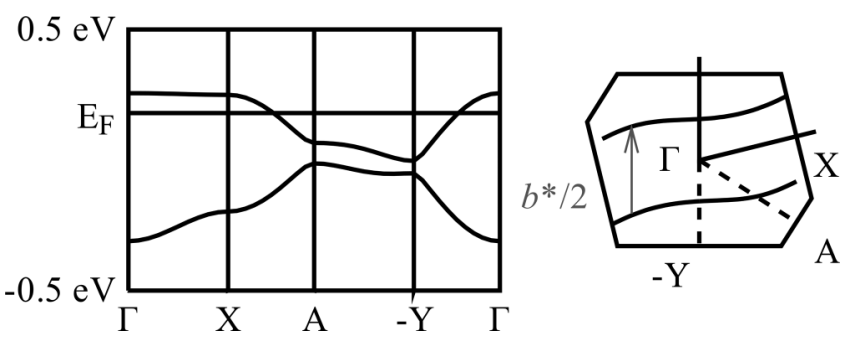

FIG. 3. Band dispersion and Fermi surface of $(\text { DMET-TTF })_{2} \mathrm{AuBr}_{2} . \boldsymbol{b}^{*}$ is a reciprocal lattice vector.

tively half filled by a dimerization gap. Due to the Q1D Fermi surface, a nesting instability with $2 k_{\mathrm{F}}$ is expected. This band structure is similar to that of (TMTCF $)_{2} X$ [19] and $(\text { DMET })_{2} \mathrm{AuI}_{2}$ [17].

\section{Resistance}

Figure 4 displays the temperature dependence of the resistivity of (DMET-TTF) ${ }_{2} \mathrm{AuBr}_{2}$ at ambient pressure. The resistivity above $26 \mathrm{~K}$ was measured using the fourprobe method and below $26 \mathrm{~K}$ using the two-probe method. There was no difference in resistivity around $26 \mathrm{~K}$ depending on both methods. The resistance shows a broad minimum around $160 \mathrm{~K}$ and drastically increases below approximately $50 \mathrm{~K}$. The broad minimum of resistance is also observed in TMTTF salts [24]. The crossover from metallic to insulating behavior with the broad minimum of resistance is thought to be due to the growth of the correlation gap in the TomonagaLuttinger liquid for a one-dimensional interacting electron system.

\section{Spin susceptibility}

We measured the temperature dependence of static susceptibility in (DMET-TTF $)_{2} \mathrm{AuBr}_{2}$ using powdered samples with a magnetic field of $2 \mathrm{~T}$ at ambient pressure. Figure 5 shows the temperature dependence of the spin susceptibility $\chi_{s}$, which was obtained by subtracting the core diamagnetism from the measured susceptibility. The core diamagnetism $\chi_{\text {dia }}$ was evaluated as $\chi_{\text {dia }}=-3.78 \mathrm{emu} / \mathrm{mol} \mathrm{f.u}$. by the susceptibilities $\chi_{\text {DMET-TTF }}=-1.07 \times 10^{-4} \mathrm{emu} / \mathrm{mol}$, $\chi_{\mathrm{TBA} \cdot \mathrm{AuBr}_{2}}=-3.03 \times 10^{-4} \mathrm{emu} / \mathrm{mol}, \quad \chi_{\mathrm{TBA} \cdot \mathrm{Br}}=-1.75 \times$ $10^{-4}$, and $\chi_{\mathrm{Br}^{-}}=-0.36 \times 10^{-4} \mathrm{emu} / \mathrm{mol}$ [25]. From 300 to around $40 \mathrm{~K}$, the spin susceptibility decreases linearly with decreasing temperature. The spin susceptibility rapidly declines with decreasing temperature below $40 \mathrm{~K}$, reaching a minimum at $22 \mathrm{~K}$. The spin susceptibility rises with decreasing temperature less than $22 \mathrm{~K}$. These properties are reminiscent of an AFM order.

TABLE II. The overlap integrals of (DMET-TTF $)_{2} \mathrm{AuBr}_{2},(\text { DMET-STF })_{2} \mathrm{AuBr}_{2}$, and $(\mathrm{TMTTF})_{2} \mathrm{Br}\left(\times 10^{-3}\right)$.

\begin{tabular}{|c|c|c|c|c|c|c|c|c|c|c|}
\hline & $S_{\mathrm{S} 1}$ & $S_{\mathrm{S} 2}$ & $S_{\mathrm{I} 1}$ & $S_{\mathrm{I} 2}$ & $S_{\mathrm{I} 3}$ & $S_{\mathrm{I} 4}$ & $S_{\mathrm{S}}$ & $S_{\mathrm{I}}$ & $S_{\mathrm{S}} / S_{\mathrm{I}}$ & $S_{\mathrm{S} 2} / S_{\mathrm{S} 1}$ \\
\hline$(\text { DMET-TTF })_{2} \mathrm{AuBr}_{2}$ & -14.27 & -11.12 & -1.15 & -1.87 & 1.34 & 0.00 & 12.69 & 1.10 & 11.5 & 0.78 \\
\hline$(\text { DMET-STF })_{2} \mathrm{AuBr}_{2}$ [18] & -24.70 & -27.95 & -2.93 & -3.69 & 1.94 & 0.00 & 26.32 & 2.37 & 11.1 & 1.13 \\
\hline$(\mathrm{TMTTF})_{2} \mathrm{Br}[21]$ & -13.55 & -12.16 & -1.03 & -0.98 & 1.57 & 0.12 & 12.85 & 1.04 & 12.3 & 0.90 \\
\hline
\end{tabular}




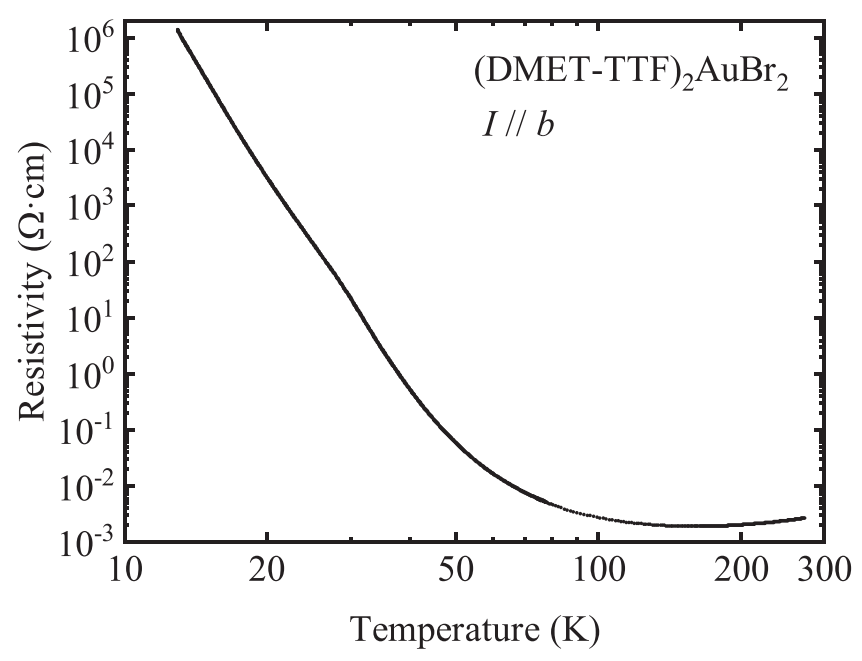

FIG. 4. Temperature dependence of the resistivity in (DMET-TTF $)_{2} \mathrm{AuBr}_{2}$. The current flows parallel to the $b$ axis.

The spin susceptibility in (TMTTF $)_{2} \mathrm{Br}$, which shows the $\mathrm{C}$-SDW transition at $14 \mathrm{~K}$, also shows the quick decline above the transition temperature and a dip structure at $14 \mathrm{~K}$ [26]. The fluctuation impact of low-dimensional phase transitions such as the charge density wave or SP is suggested to cause this quick decrease. Furthermore, a rapid decrease in spin susceptibility slightly above the transition temperature has been observed in (TMTTF $)_{2} \mathrm{SbF}_{6}$, which shows the AFM transition at $7 \mathrm{~K}$ in the $\mathrm{CO}$ phase, and in (TMTTF) ${ }_{2} \mathrm{SCN}$, which shows the AFM transition at $8 \mathrm{~K}$ in the anion-ordered phase [27]. However, the spin susceptibility in (TMTSF) ${ }_{2} \mathrm{PF}_{6}$, which shows a metal-insulator (IC-SDW) transition at $12 \mathrm{~K}$, does not show a rapid decrease just above the transition temperature [28], whereas (TMTTF $)_{2} \mathrm{Br}$ (C-SDW) shows a rapid decrease as described above. At high temperatures, the spin susceptibility is described by the Bonner-Fisher type temperature dependence [29]. The spin susceptibility of (DMET-TTF $)_{2} \mathrm{AuBr}_{2}$ is also thought to be described by

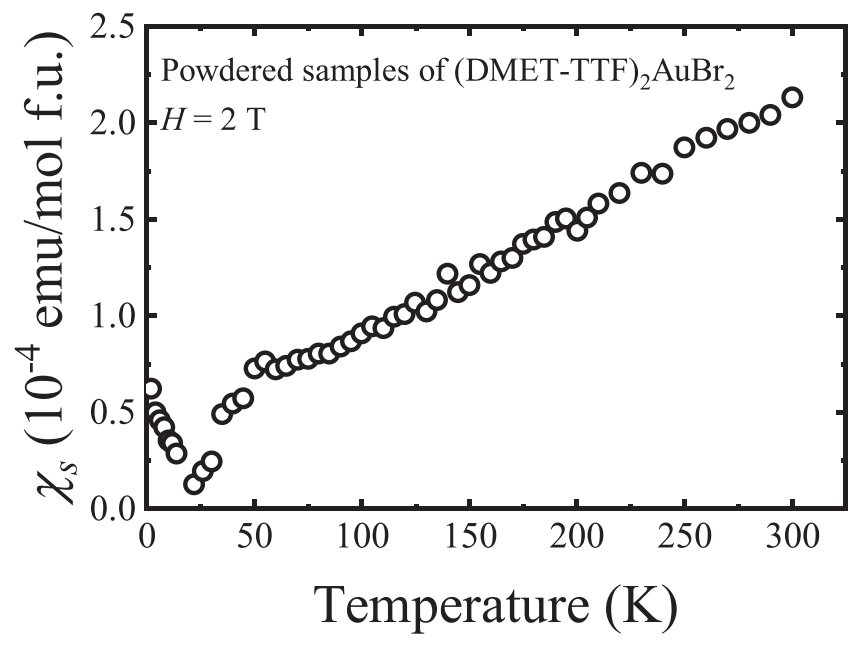

FIG. 5. Temperature dependence of the spin susceptibility in $(\mathrm{DMET}-\mathrm{TTF})_{2} \mathrm{AuBr}_{2}$ measured on a powdered sample. Core diamagnetism was subtracted from the original data.

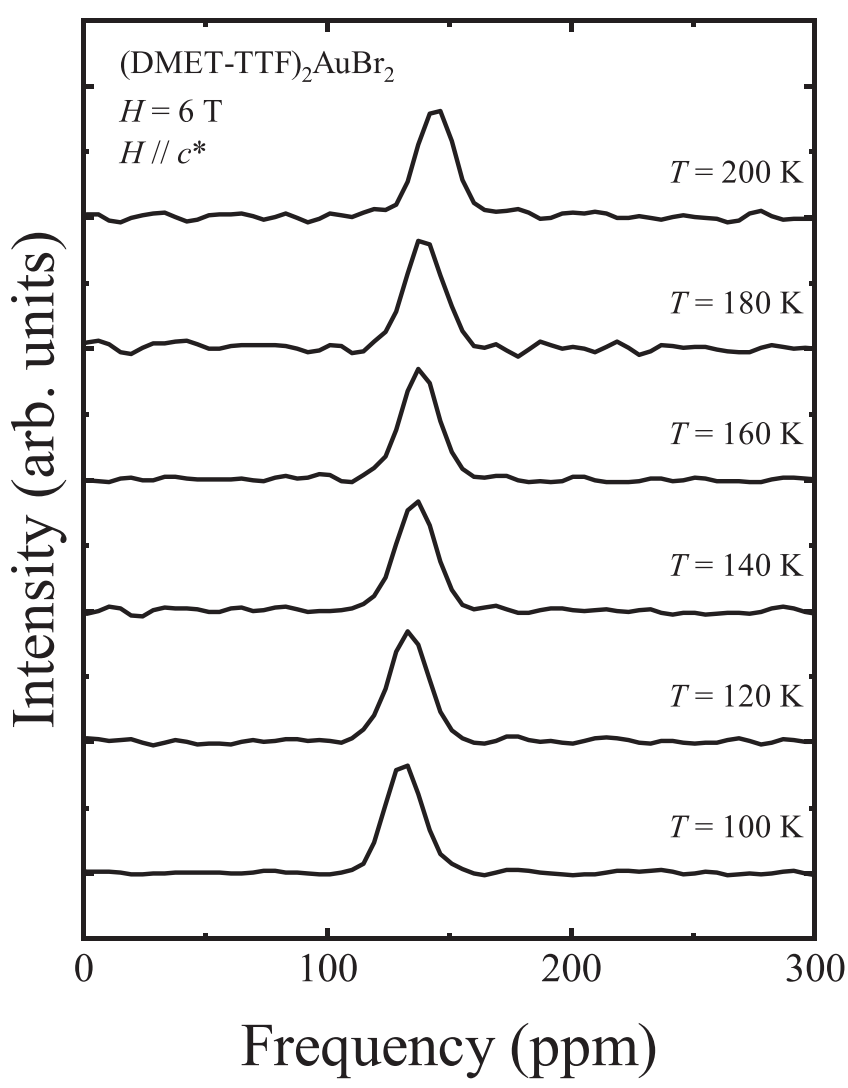

FIG. 6. ${ }^{13} \mathrm{C}-\mathrm{NMR}$ spectra in the paramagnetic phase of (DMET-TTF $)_{2} \mathrm{AuBr}_{2}$. The magnetic field corresponding to 65.11 MHz was applied along the $c^{*}$ axis.

the spin-1/2 Heisenberg AFM model. The absolute value is smaller than (TMTTF $)_{2} \mathrm{Br}$, and the small spin susceptibility suggests the large exchange coupling $J$ in the spin-1/2 Heisenberg AFM model framework.

\section{E. ${ }^{13} \mathrm{C}$ NMR in the magnetic ordering phase}

To verify the magnetic ordering state, we measured the NMR spectra at $65.11 \mathrm{MHz}$ in (DMET-TTF) ${ }_{2} \mathrm{AuBr}_{2}$ below $200 \mathrm{~K}$. A magnetic field of $6 \mathrm{~T}$ was applied along with the $c^{*}$ axis corresponding to the normal of the $a b$ plane. Figure 6 shows the NMR spectra in the paramagnetic region. In (DMET-TTF $)_{2} \mathrm{AuBr}_{2}$, there is one peak at the crystallographic ${ }^{13} \mathrm{C}$ site in a unit cell and one peak was observed in the paramagnetic phase. As the temperature decreases, the peak's NMR shift slightly decreases. As shown in Fig. 7, a peak in the paramagnetic phase splits into three peaks at $22 \mathrm{~K}$, at which a dip structure in the spin susceptibility was observed, and the central peak diminishes down to $17 \mathrm{~K}$. With decreasing temperature, the split width between the lower and higher peaks increases. These behaviors are considered a requirement of AFM order.

In addition, we measured the spin-lattice relaxation rate $T_{1}^{-1}$ in a magnetic field of $6 \mathrm{~T}$ applied in the direction of the $c^{*}$ axis. Figure 8 presents the temperature dependence of $T_{1}^{-1}$. $T_{1}^{-1}$ has a small temperature dependence in the paramagnetic phase and shows a sharp divergent peak around $22 \mathrm{~K}$. This divergent behavior of $T_{1}^{-1}$ supports the AFM transition. Below 


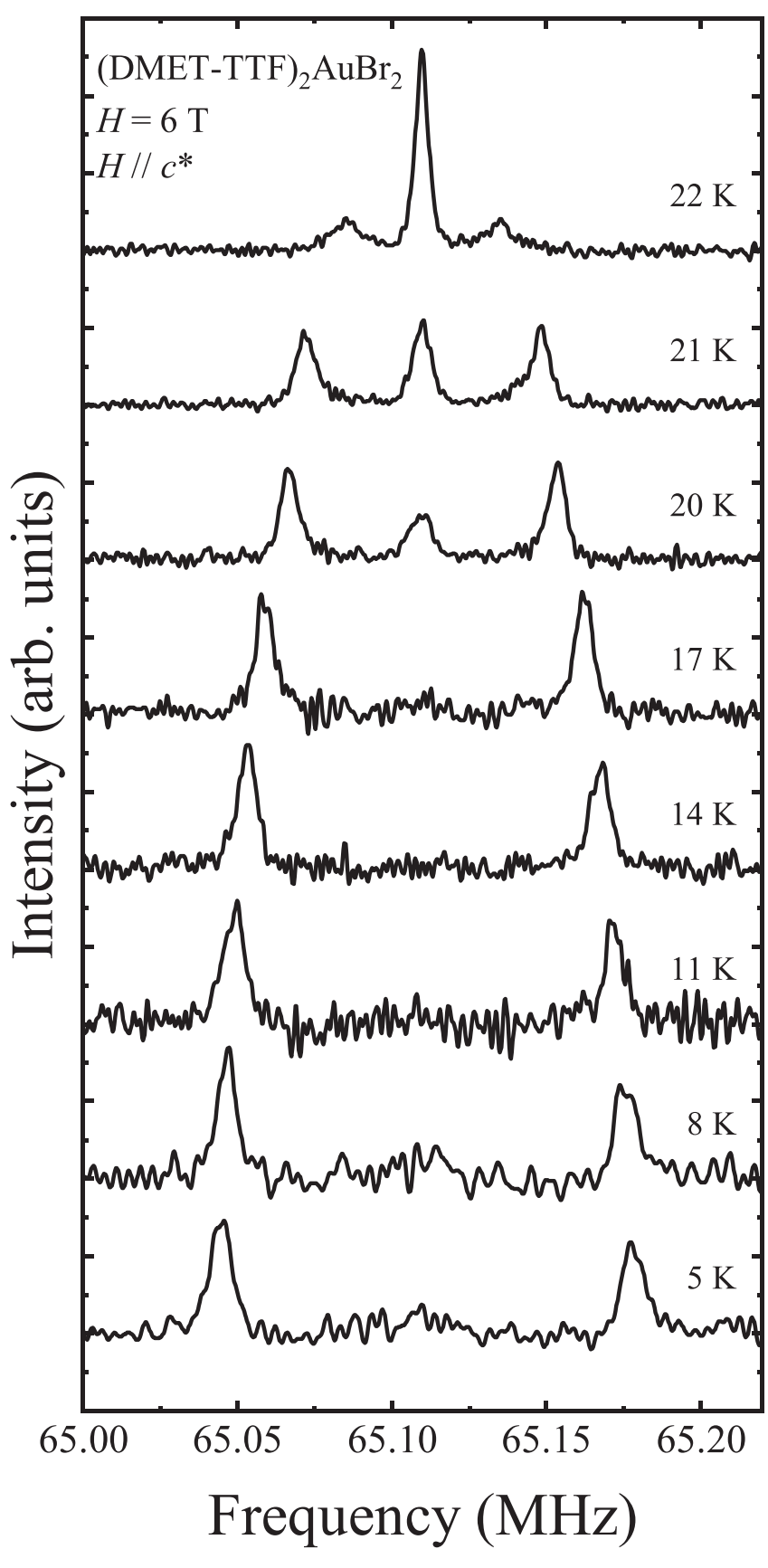

FIG. 7. ${ }^{13} \mathrm{C}-\mathrm{NMR}$ spectra in (DMET-TTF $)_{2} \mathrm{AuBr}_{2}$ at various temperatures. A magnetic field corresponding to $65.11 \mathrm{MHz}$ was applied along the $c^{*}$ axis.

$22 \mathrm{~K}, T_{1}^{-1}$ of both the lower and higher peaks declines exponentially with decreasing temperature in the same manner. These results indicate that the commensurate AFM transition occurs at $22 \mathrm{~K}$.

A simple explanation of the peak splitting is the up-down spin structure due to the dimer Mott insulating state. The AFM state of the dimer Mott insulator with 2:1 stoichiometry has the amplitude of the magnetic moment as $m=1 \mu_{B} /$ dimer. The dimer Mott insulators $\beta^{\prime}$-(BEDT-TTF $)_{2} \mathrm{ICl}_{2}[30,31]$ and $(\mathrm{TMTTF})_{2} \mathrm{SbF}_{6}[5,32]$ at ambient pressure show $1 \mu_{B} /$ dimer in the AFM phase. However, C-SDW and IC-SDW phases

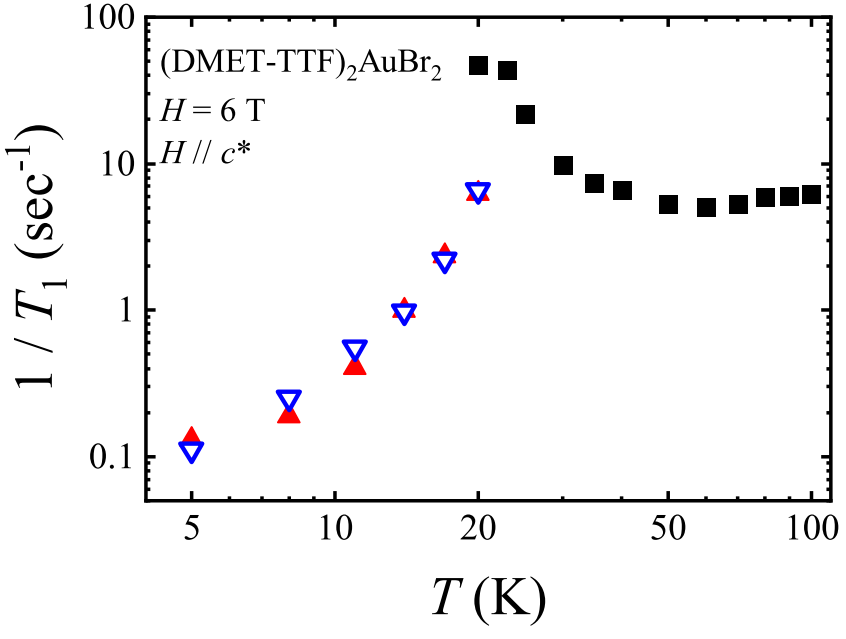

FIG. 8. Temperature dependence of $1 / T_{1}$ in (DMET-TTF $)_{2} \mathrm{AuBr}_{2}$. A magnetic field was applied along the $c^{*}$ axis. The open and solid triangles correspond to the lower and higher peaks in the NMR shift.

of TMTCF families have a small amplitude of the magnetic moment. To confirm "experimentally" which is suitable, we determined the hyperfine coupling tensor and estimated the amplitude of the magnetic moment in the AFM phase of (DMET-TTF $)_{2} \mathrm{AuBr}_{2}$ using the hyperfine coupling tensor.

\section{F. Hyperfine coupling and chemical shift tensor}

We determined the hyperfine coupling tensor $\mathbf{A}$ and the chemical shift tensor $\sigma$ to estimate the amplitude of the magnetic moment in the AFM phase. The NMR shift $\delta$ is represented as $\delta=K+\sigma=a \chi_{s}+\sigma$, where $K$ is the Knight shift, $a$ is the hyperfine coupling constant, and $\sigma$ is the chemical shift. In an anisotropic case, $a$ and $\sigma$ depend on the direction of the field, $H(\theta, \phi), a(\theta, \phi)$ and $\sigma(\theta, \phi)$ are expressed as $a(\theta, \phi)=\tilde{\mathbf{h}} \mathbf{A h}, \sigma(\theta, \phi)=\tilde{\mathbf{h}} \sigma \mathbf{h}$, where $\mathbf{h}$ is the directional cosine of the magnetic field. The definitions of the field angle $\theta$ and $\phi$ versus the $a, b^{\prime}$, and $c^{*}$ axes are shown in the inset of Fig. 9, where the $b^{\prime}$ axis is the $c^{*} \times a$ direction. Figure 9 displays a $\delta-\chi_{s}$ plot in (DMET-TTF $)_{2} \mathrm{AuBr}_{2}$ at various field angles. We can obtain $a(\theta, \phi)$ as a slope of the linear function and $\sigma(\theta, \phi)$ as an intercept. $\mathbf{A}$ and $\sigma$ are evaluated from a set of $a(\theta, \phi)$ and $\sigma(\theta, \phi)$,

$$
\begin{aligned}
\mathbf{A} & =\left(\begin{array}{lll}
A_{a a} & A_{a b^{\prime}} & A_{a c^{*}} \\
A_{a b^{\prime}} & A_{b^{\prime} b^{\prime}} & A_{b^{\prime} c^{*}} \\
A_{a c^{*}} & A_{b^{\prime} c^{*}} & A_{c^{*} c^{*}}
\end{array}\right) \\
= & \left(\begin{array}{rrr}
-0.03(6) & -2.16(9) & 0.34(9) \\
-2.16(9) & 7.8(2) & -3.0(4) \\
0.34(9) & -3.0(4) & 1.11(10)
\end{array}\right) \quad\left(\mathrm{kOe} / \mu_{B}\right), \\
& \boldsymbol{\sigma}=\left(\begin{array}{lll}
\sigma_{a a} & \sigma_{a b^{\prime}} & \sigma_{a c^{*}} \\
\sigma_{a b^{\prime}} & \sigma_{b^{\prime} b^{\prime}} & \sigma_{b^{\prime} c^{*}} \\
\sigma_{a c^{*}} & \sigma_{b^{\prime} c^{*}} & \sigma_{c^{*} c^{*}}
\end{array}\right) \\
& =\left(\begin{array}{rrr}
127(2) & -19(2) & 13(2) \\
-19(2) & 217(5) & -71(9) \\
13(22) & -71(9) & 99(3)
\end{array}\right) \quad(\mathrm{ppm}) .
\end{aligned}
$$




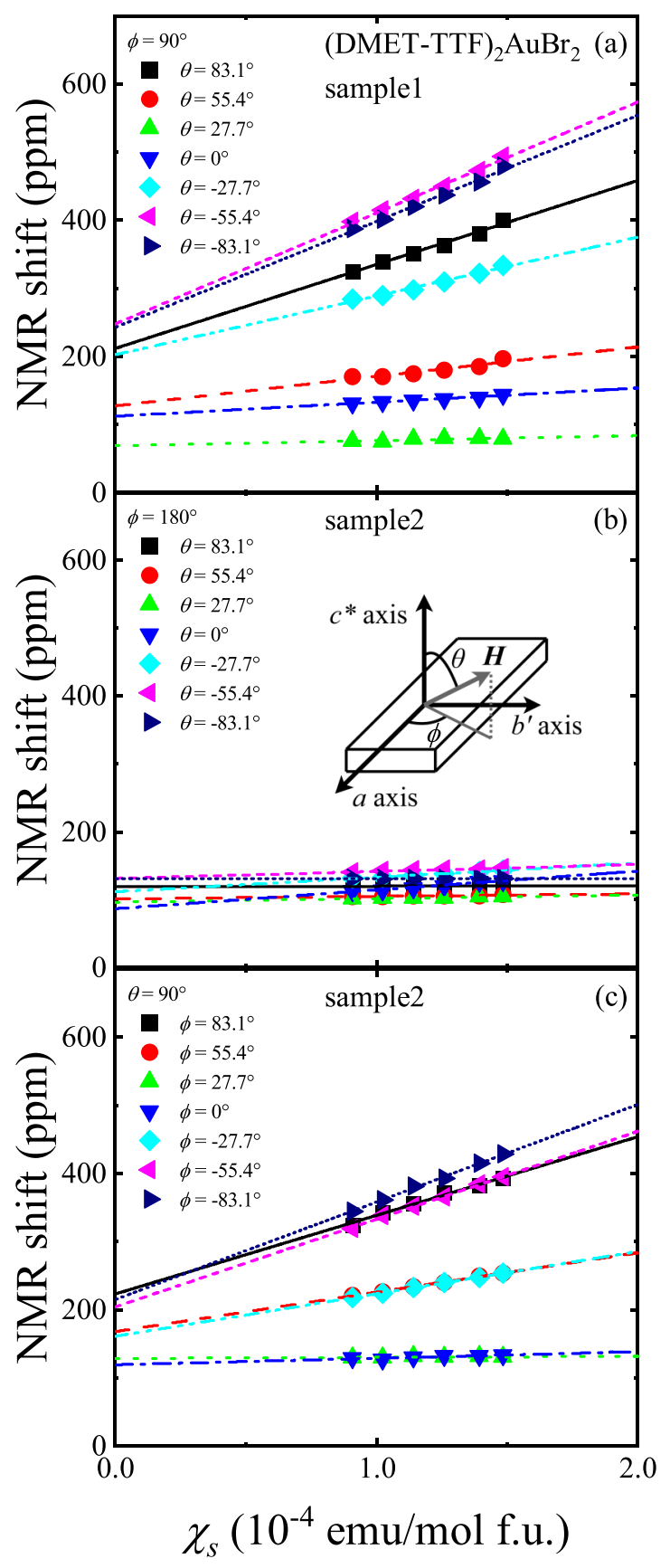

FIG. 9. $\delta-\chi_{s}$ plot in the nonmagnetic phase of (DMET-TTF) ${ }_{2} \mathrm{AuBr}_{2}$ at various field angles. (a) $\phi=90^{\circ}$, (b) $\phi=180^{\circ}$, and (c) $\theta=90^{\circ}$. The temperature range is between 100 and $200 \mathrm{~K}$. Inset in (b): Sample and $\left(a, b^{\prime}, c^{*}\right)$ axis configuration of the measurement.

The elements of hyperfine coupling and the chemical shift tensor are semiquantitatively similar to (TMTTF $)_{2} \mathrm{Br}[5]$ and $(\text { TMTSF })_{2} \mathrm{PF}_{6}[33]$.

\section{G. Estimation of the amplitude of magnetic moment}

We measured the angular field dependence of the NMR shift around the $a$ axis at $14 \mathrm{~K}$ to estimate the amplitude of the magnetic moment in the antiferromagnetic phase in (DMET-TTF $)_{2} \mathrm{AuBr}_{2}$, as shown in Fig. 10(a). A magnetic
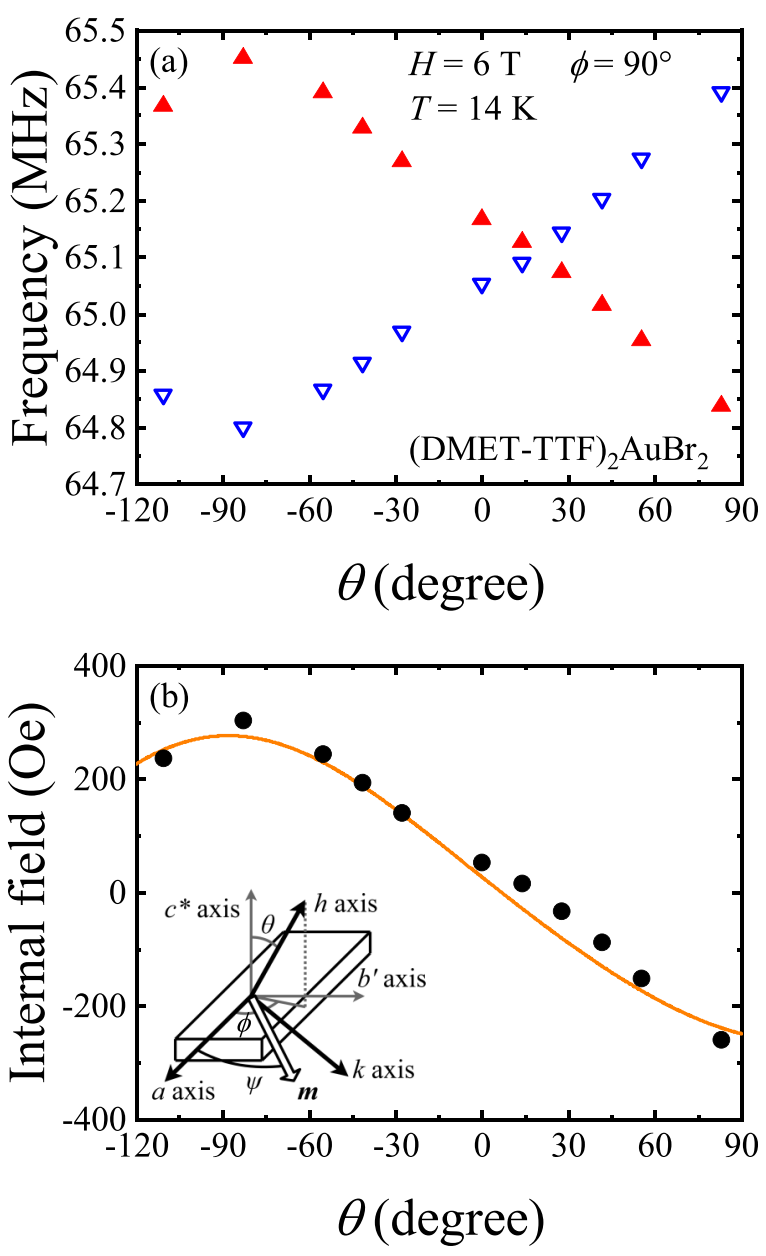

FIG. 10. (a) Field angle dependence of NMR shift at $14 \mathrm{~K}$ in (DMET-TTF $)_{2} \mathrm{AuBr}_{2}$. The open and solid triangles correspond to the up and down spins. (b) Angular dependence of an internal field at $14 \mathrm{~K}$ in (DMET-TTF) ${ }_{2} \mathrm{AuBr}_{2}$. The solid curve is the fitting curve with parameters of $m=0.061 \mu_{B} /$ molecule and $\psi=2^{\circ}$. Inset: Sample and $\left(a, b^{\prime}, c^{*}\right)$ and $(a, k, h)$ axis measurement configuration.

field is applied perpendicular to the $a$ axis. The frequency shifts of two peaks show an opposite angular dependence. Therefore, we can classify the NMR peaks as contributing to each of the two local moments [the open and solid triangles in Fig. 10(a)]. Figure 10(b) shows half of the difference in the NMR shift of the two peaks' frequency divided by the gyromagnetic ratio. Here, the chemical shift is canceled, and the difference corresponds only to the internal field.

Generally, the antiferromagnetic moments of organic conductors in the high field are expected to flop perpendicular to the external field of $6 \mathrm{~T}$. Hence, the direction of the moment is in the $a k$ plane shown in the inset of Fig. 10(b), where the $h$ axis is the external field direction, and the $k$ axis is the $h \times a$ direction. In this condition, the rotating angle corresponds to the $\theta$ with $\phi=90^{\circ}$. We calculate the hyperfine coupling tensor $\mathbf{A}^{\prime}$ in the $(a, k, h)$ coordinates using a rotational matrix $\mathbf{U}$, as $\left(\mathbf{U A U}^{-\mathbf{1}}\right)$. We can set the moment as a function of angle $\psi$ from the $a$ axis; $\mathbf{m}^{\prime}=(m \cos \psi, m \sin \psi, 0)$, and the internal field is expressed as

$$
H_{\text {int }}=\left(\mathbf{U A U}^{-\mathbf{1}}\right)_{a h} m \cos \psi+\left(\mathbf{U A U}^{\mathbf{- 1}}\right)_{k h} m \sin \psi,
$$


where

$$
\begin{aligned}
\left(\mathbf{U A U}^{-1}\right)_{a h} & =A_{a b^{\prime}} \sin \theta+A_{a c^{*}} \cos \theta, \\
\left(\mathbf{U A U}^{-1}\right)_{k h} & =\frac{1}{2} A_{b^{\prime} b^{\prime}} \sin 2 \theta+A_{b^{\prime} c^{*}} \cos 2 \theta-\frac{1}{2} A_{c^{*} c^{*}} \sin 2 \theta .
\end{aligned}
$$

The solid curve in Fig. 10(b) is the fitting curve with parameters of $m=0.061 \mu_{B} /$ molecule and $\psi=2^{\circ}$. The magnetic moment is approximately parallel to the $a$ axis. The $a$ axis is expected to be the easy axis because the direction is invariant while the magnetic field is applied in the $b^{\prime} c^{*}$ plane.

We estimated the amplitude of the magnetic moment as $m=0.06 \mu_{B} /$ molecule at $14 \mathrm{~K}$, whereas the amplitude is expected to be $0.5 \mu_{B} /$ molecule in an AFM state of the dimer Mott insulator. It is comparable to the SDW amplitude in (TMTTF $)_{2} \mathrm{Br}$ reported as $0.11 \mu_{B} /$ molecule [5] by ${ }^{13} \mathrm{C}$ NMR, $0.14 \mu_{B} /$ molecule [4] by ${ }^{1} \mathrm{H}$ NMR, in (TMTSF) ${ }_{2} \mathrm{PF}_{6}$ reported as $0.08 \mu_{B} /$ molecule [34] by ${ }^{1} \mathrm{H}$ $\mathrm{NMR}$, and in (DMET-STF $)_{2} \mathrm{Au}(\mathrm{CN})_{2}$ reported as the order of $0.1 \mu_{B} /$ molecule [35]. From the small amplitude and splitting peaks, the ground state of (DMET-TTF) ${ }_{2} \mathrm{AuBr}_{2}$ at ambient pressure is thought to be the C-SDW phase. The dimer Mott insulator model well explains the CO, SP, and AFM phases in Q1D organic conductor TMTCF families [36]. However, the SDW phase in TMTCF families is located at the boundary of the metal-insulator transition and forms the C-SDW phase on the insulator side and the IC-SDW phase on the metallic side [37]. The SDW transition temperature shows a maximum at the boundary of the MI transition. The IC-SDW on the metallic side is well explained by the nesting model of the Q1D Fermi surface with $2 k_{\mathrm{F}}$ instability.

To verify the above interpretation, it is important whether this salt is located at the metal-insulator boundary. We found that (DMET-TTF) ${ }_{2} \mathrm{AuBr}_{2}$ shows a metal-insulator transition at $32 \mathrm{~K}$ under $0.3 \mathrm{GPa}$, and at $13 \mathrm{~K}$ under $0.5 \mathrm{GPa}$ with the emergence of nonlinear conductivity and an isostructural salt (DMET-TTF) ${ }_{2} \mathrm{I}_{3}$ shows a metal-insulator (SDW) transition at $40 \mathrm{~K}$ at ambient pressure [10]. These results are reminiscent of those of (TMTTF $)_{2} \mathrm{Br}$. (TMTTF) $)_{2} \mathrm{Br}$ is located at the boundary of the metal-insulator transition. Therefore, we conclude that (DMET-TTF $)_{2} \mathrm{AuBr}_{2}$ is located at the boundary of the metal-insulator transition. The SDW phase of the TMTCF family is composed of the C-SDW and IC-SDW phases and the IC-SDW phase is well explained by the $2 k_{\mathrm{F}}$ instability model. Therefore, C-SDW on (DMET-TTF) ${ }_{2} \mathrm{AuBr}_{2}$ can be explained by the $2 k_{\mathrm{F}}$ instability model in the CL state.

Figure 11 shows the temperature dependence of the magnetic moment. As temperature decreases, the magnetic moment increases by approximately $17 \%$ at $5 \mathrm{~K}$ from a value of $14 \mathrm{~K}$. We can fit the magnetic moment using the critical exponent formula $M(T) \propto\left[1-\left(T / T_{\mathrm{SDW}}\right)\right]^{\beta}$ and obtain $\beta=0.21$.

\section{H. The magnetic structure in the C-SDW phase}

We found that (DMET-TTF) ${ }_{2} \mathrm{AuBr}_{2}$ displays the C-SDW phase below $22 \mathrm{~K}$. The SDW structure is assumed to be $(\uparrow \downarrow \uparrow$ $\downarrow)$ or ( $\uparrow \downarrow \downarrow)$ along the $b$ axis since there are up and down moments in the C-SDW phase. The SDW state is stabilized by the nesting instability of a Fermi surface, and SDW formation depends on a nesting vector. In (TMTSF) ${ }_{2} \mathrm{PF}_{6}$, the SDW wave

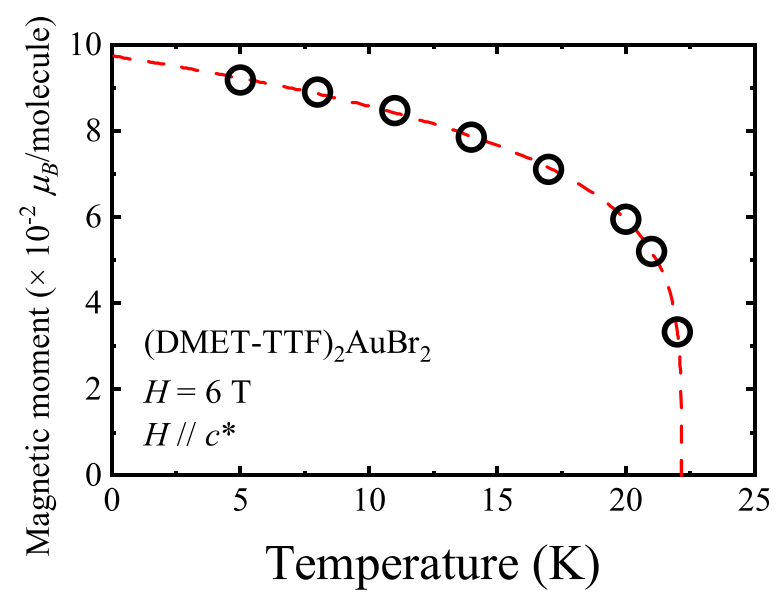

FIG. 11. Temperature dependence of the magnetic moment of the SDW state. The dashed curve is the fitting curve of the critical exponent formula $M(T) \propto\left[1-\left(T / T_{\mathrm{SDW}}\right)\right]^{\beta}$ with parameters of $\beta=0.21$.

vector along the stacking direction was estimated $\pi / a$ by ${ }^{1} \mathrm{H}-$ NMR measurements, which is consistent with the expected nesting vector of the Q1D Fermi surface [34]. Estimation of the SDW wave number by ${ }^{1} \mathrm{H}-\mathrm{NMR}$ measurements is also performed in (TMTTF) ${ }_{2} \mathrm{Br}$ [4]. Although the CL state was observed just above the C-SDW transition, the C-SDW state was thought to stabilize by the $2 k_{\mathrm{F}}$ instability. Notably, the $2 k_{\mathrm{F}}$ instability was reported above the C-SDW transition in $(\mathrm{TMTTF})_{2} \mathrm{Br}$ by x-ray diffuse scattering [38]. Therefore, the $2 k_{\mathrm{F}}$ instability was also expected in (DMET-TTF) $)_{2} \mathrm{AuBr}_{2}$. Indeed, as shown in Fig. 3, a nesting vector between two open Fermi surfaces is approximately $\boldsymbol{b}^{*} / 2$, where $\boldsymbol{b}^{*}$ is a reciprocal lattice vector. As a result, the expected spin stricture of the C-SDW phase in (DMET-TTF) $)_{2} \mathrm{AuBr}_{2}$ is $(\uparrow \uparrow \downarrow \downarrow)$ along the $b$ axis as shown in Fig. 12. However, the spin structure of the C-SDW phase in (TMTTF) $)_{2} \mathrm{Br}$ was assigned as $(\uparrow \circ \downarrow \circ)$ by ${ }^{1} \mathrm{H}$ - and ${ }^{13} \mathrm{C}$-NMR measurements $[4,5]$. Theoretically, the $(\uparrow \uparrow \downarrow \downarrow)$ configuration is energetically stable due to the exchange interaction [39]. The C-SDW in $(\mathrm{TMTTF})_{2} \mathrm{Br}$ appears adjacent to the $\mathrm{CO}$ phase, in which the $(\uparrow \circ \downarrow \circ)$ type of spin alignment was expected from the universal phase diagram of TMTCF families. Therefore, the $(\uparrow \circ \downarrow \circ)$ configuration in (TMTTF) $)_{2} \mathrm{Br}$ appears due to $\mathrm{CO}$ instability. It is theoretically suggested that the CO phase in the Q1D system is suppressed by dimerization [39,40]. Since (DMET-TTF) ${ }_{2} \mathrm{AuBr}_{2}$ has a strong dimerization and $\mathrm{CO}$ anomalies were not observed in the paramagnetic state, the theoretically predicted $(\uparrow \uparrow \downarrow \downarrow)$ configuration in the C-SDW phase of (DMET-TTF $)_{2} \mathrm{AuBr}_{2}$ is thought to be stabilized without the $\mathrm{CO}$ effect. (DMET-TTF) ${ }_{2} \mathrm{AuBr}_{2}$

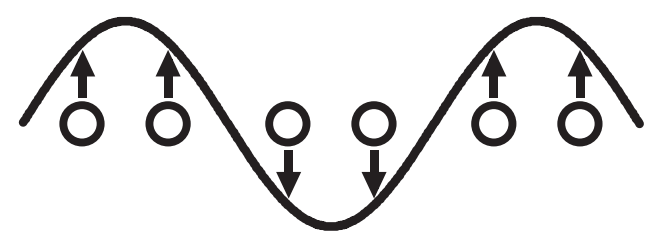

FIG. 12. SDW structure in (DMET-TTF $)_{2} \mathrm{AuBr}_{2}$. 
is the first $(\uparrow \uparrow \downarrow \downarrow)$ type C-SDW in the Q1D organic system.

\section{Comparison with (DMET-STF) $)_{2} \mathrm{AuBr}_{2}$}

We compare with the isostructural material (DMET-STF $)_{2} \mathrm{AuBr}_{2}$. There are two types of overlaps ( $\mathrm{S} 1$ and $\mathrm{S} 2$ ) between the neighboring molecules even though the two independent interplanar distances $(3.54 \AA$ for $\mathrm{S} 1$ and $3.55 \AA$ for S2) in (DMET-STF) $)_{2} \mathrm{AuBr}_{2}$ are almost equivalent. In (DMET-STF) ${ }_{2} \mathrm{AuBr}_{2}$, Se-Se contacts become significant for the overlap integrals because the van der Waals radius of the Se atom is larger than that of the $\mathrm{S}$ atom. The S2 type of overlap has two Se-Se contacts shorter than the van der Waals radii sum. However, the S1 type of overlap has no shorter Se-Se contact $[18,41]$. As listed in Table II, the overlap integrals in (DMET-STF) ${ }_{2} \mathrm{AuBr}_{2}$ at ambient pressure are $S_{\mathrm{S} 1}=-0.02470, S_{\mathrm{S} 2}=-0.02795$, and the effective dimerization of the overlap integrals $S_{\mathrm{S} 2} / S_{\mathrm{S} 1}$ in (DMET-STF) $)_{2} \mathrm{AuBr}_{2}$ is 1.13 , suggesting that (DMET-STF $)_{2} \mathrm{AuBr}_{2}$ is also the dimerized Q1D system, although the magnitude relationship between $\mathrm{S} 1$ and $\mathrm{S} 2$ is reversed. We found that DMET system is equivalent to the Q1D system as well as the TMTCF system and has a larger dimerization than the TMTCF system. In TMTCF families, the C-SDW phase becomes the superconducting phase through the IC-SDW phase by applying pressure. It is interesting to investigate how the C-SDW phase without $\mathrm{CO}$ instability changes under pressure.

We are planning the transport and ${ }^{13} \mathrm{C}-\mathrm{NMR}$ measurements in (DMET-TTF $)_{2} \mathrm{AuBr}_{2}$ under pressure to clarify the applicability of the universal phase diagram depending on physical and chemical pressure on DMET families with linear anions such as the case of TMTCF families.

\section{CONCLUSION}

We synthesized a Q1D organic conductor (DMET-TTF) $)_{2} \mathrm{AuBr}_{2}$ and calculated the Q1D Fermi surface using the Hückel tight-binding approximation. As a result, (DMET-TTF $)_{2} \mathrm{AuBr}_{2}$ was found to be a strong dimerized system compared to TMTCF families. The resistance shows a broad minimum around $160 \mathrm{~K}$ and insulator behavior below this temperature, as with (TMTTF $)_{2} \mathrm{Br}$. The susceptibility, the NMR spectra, and $T_{1}$ suggest the AFM transition occurs at $22 \mathrm{~K}$. Using the hyperfine coupling and chemical shift tensors to the angle dependence of the NMR shift in the AFM phase, we revealed the C-SDW state with up and down spins having $0.06 \mu_{B} /$ molecule at $14 \mathrm{~K}$. By considering the $2 k_{\mathrm{F}}$ instability of the Fermi surface, we concluded that the expected spin structure is $(\uparrow \uparrow \downarrow \downarrow)$ type which is stabilized by the strong dimerization.

\section{ACKNOWLEDGMENTS}

We would like to thank Professor S. Noro and Dr. K. Takahashi of Hokkaido University for the X-ray diffraction analysis and Professor H. Yoshino of Osaka City University for valuable discussions. This work was supported by the Japan Society for the Promotion of Science KAKENHI (Grant No. 19K0370709), and Hokkaido University (JP), Global Facility Center (GFC), Advanced Physical Property Open Unit (APPOU), funded by MEXT (JP) under "Support Program for Implementation of New Equipment Sharing System" (JPMXS0420100318).
[1] D. Jérome, A. Mazaud, M. Ribault, and K. Bechgaard, J. Phys. Lett. 41, 95 (1980).

[2] D. Jérome, Science 252, 1509 (1991).

[3] E. Barthel, G. Quirion, P. Wzietek, D. Jérome, J. B. Christensen, M. Jørgensen, and K. Bechgard, Europhys. Lett. 21, 87 (1993).

[4] T. Nakamura, T. Nobutoki, Y. Kobayashi, T. Takahashi, and G. Saito, Synth. Met. 70, 1293 (1995).

[5] S. Hirose, Y. Liu, and A. Kawamoto, Phys. Rev. B 88, 125121 (2013).

[6] K. Kikuchi, M. Kikuchi, T. Namiki, K. Saito, I. Ikemoto, K. Murata, T. Ishiguro, and K. Kobayashi, Chem. Lett. 16, 931 (1987).

[7] K. Kikuchi, K. Murata, Y. Honda, T. Namiki, K. Saito, H. Anzai, K. Kobayashi, T. Ishiguro, and I. Ikemoto, J. Phys. Soc. Jpn. 56, 4241 (1987).

[8] K. Kikuchi, K. Murata, Y. Honda, T. Namiki, K. Saito, T. Ishiguro, K. Kobayahsi, and I. Ikemoto, J. Phys. Soc. Jpn. 56, 3436 (1987).

[9] K. Kikuchi, Y. Honda, Y. Ishikawa, K. Saito, I. Ikemoto, K. Murata, H. Anzai, T. Ishiguro, and K. Kobayashi, Solid State Commun. 66, 405 (1988)

[10] K. Saito, A. Sato, K. Kikuchi, H. Nishikawa, I. Ikemoto, and M. Sorai, J. Phys. Soc. Jpn. 69, 3602 (2000).
[11] N. Svenstrup, K. M. Rasumussen, T. K. Hansen, and J. Becher, Synthesis 1994, 809 (1994).

[12] S. Hirose, M. Misawa, and A. Kawamoto, Crystals 2012, 1034 (2012).

[13] M. C. Burla, M. Camalli, B. Carrozzini, G. L. Cascarano, C. Giacovazzo, G. Polidori, and R. Spagna, J. Appl. Crystallogr. 36, 1103 (2003).

[14] G. M. Sheldrick, Acta Crystallogr., Sect. A 64, 112 (2008).

[15] C. F. Macrae, I. Sovago, S. J. Cottrell, P. T. A. Galek, P. McCabe, E. Pidcock, M. Platings, G. P. Shields, J. S. Stevens, M. Towler, and P. A. Wood, J. Appl. Crystallogr. 53, 226 (2020).

[16] T. Mori, A. Kobayashi, Y. Sasaki, H. Kobayashi, G. Saito, and H. Inokuchi, Bull. Chem. Soc. Jpn. 57, 627 (1984).

[17] R. Kato, S. Aonuma, Y. Okano, H. Sawa, M. Tamura, M. Kinoshita, K. Oshima, A. Kobayashi, K. Bun, and H. Kobayashi, Synth. Met. 61, 199 (1993).

[18] Y. Ishikawa, M.Sc. thesis, Tokyo Metropolitan University, 1989.

[19] P. M. Grant, J. Phys. (Paris), Colloq. 44, C3-847 (1983).

[20] T. Ishiguro, K. Yamaji, and G. Saito, Organic Superconductors, 2nd ed. (Springer, Berlin, 1998), Chaps. 3 and 4.

[21] J. L. Galigné, B. Liautard, S. Peytavin, G. Brun, J. M. Fabre, E. Torreilles, and L. Giral, Acta Crystallogr., Sect. B 34, 620 (1978). 
[22] O. V. Dolomanov, L. J. Bourhis, R. J. Gildea, J. A. K. Howard, and H. Puschmann, J. Appl. Crystallogr. 42, 339 (2009).

[23] L. Ducasse, M. Abderrabba, J. Hoarau, M. Pesquer, B. Gallois, and J. Gaultier, J. Phys. C 19, 3805 (1986).

[24] C. Coulon, P. Delhaes, S. Flandrois, R. Lagnier, E. Bonjour, and J. M. Fabre, J. Phys. (Paris) 43, 1059 (1982).

[25] Nihon-Kagakukai, Kagaku Binran (Kiso-hen) V (Maruzen, Tokyo, 1993) (in Japanese).

[26] N. Matsunaga, K. Nomura, T. Nakamura, T. Takahashi, G. Saito, S. Takasaki, J. Yamada, S. Nakatsuji, and H. Anzai, Physica B 284-288, 1583 (2000).

[27] B. Salameh, S. Yasin, M. Dumm, G. Untereiner, L. Montgomery, and M. Dressel, Phys. Rev. B 83, 205126 (2011)

[28] K. Mortensen, Y. Tomkiewicz, and K. Bechgaard, Phys. Rev. B 25, 3319 (1982).

[29] M. Dumm, A. Loidl, B. W. Fravel, K. P. Starkey, L. K. Montgomery, and M. Dressel, Phys. Rev. B 61, 511 (2000).

[30] H. Taniguchi, M. Miyashita, K. Uchiyama, K. Satoh, N. Mori, H. Okamoto, K. Miyagawa, K. Kanoda, M. Hedo, and Y. Uwatoko, J. Phys. Soc. Jpn. 72, 468 (2003).

[31] Y. Eto and A. Kawamoto, Phys. Rev. B 81, 020512(R) (2010).
[32] N. Matsunaga, S. Hirose, N. Shimohara, T. Satoh, T. Isome, M. Yamomoto, Y. Liu, A. Kawamoto, and K. Nomura, Phys. Rev. B 87, 144415 (2013).

[33] Y. Kimura, M. Misawa, and A. Kawamoto, Phys. Rev. B 84, 045123 (2011).

[34] T. Takahashi, Y. Maniwa, H. Kawamura, and G. Saito, Physica (Amsterdam) 143, 417 (1986).

[35] K. Kanoda, T. Takahashi, T. Tokiwa, K. Kikuchi, K. Saito, I. Ikemoto, and K. Kobayashi, Phys. Rev. B 38, 39 (1988).

[36] K. Yoshimi, H. Seo, S. Ishibashi, and S. E. Brown, Phys. Rev. Lett. 108, 096402 (2012).

[37] B. J. Klemme, S. E. Brown, P. Wzietek, G. Kriza, P. Batail, D. Jérome, and J. M. Fabre, Phys. Rev. Lett. 75, 2408 (1995).

[38] J. P. Pouget and S. Ravy, Synth. Met. 85, 1523 (1997).

[39] H. Seo and H. Fukuyama, J. Phys. Soc. Jpn. 66, 1249 (1997).

[40] M. Tsuchiizu, H. Yoshioka, and Y. Suzumura, J. Phys. Soc. Jpn. 70, 1460 (2001).

[41] K. Kikuchi, Y. Ishikawa, K. Saito, I. Ikemoto, and K. Kobayashi, Acta Crystallogr., Sect. C 44, 466 (1988). 